\title{
A step forward in the use of SPECT imaging with I-123 MIBG
}

\author{
Mouhamad Abdallah, MD, and Myron C. Gerson, MD
}

\section{See related article, pp. 92-99}

With the publication of the AdreView Myocardial Imaging for Risk Evaluation in Heart Failure Study (ADMIRE-HF), it has become clear that a simple ratio of heart-to-mediastinum (H/M) activity of I-123 metaiodobenzylguanidine (MIBG), on an anterior planar image is a strong predictor of cardiac prognosis in heart failure patients with a reduced left ventricular ejection fraction and New York Heart Association class II or III symptoms. The planar I-123 MIBG H/M ratio provides predictive information for outcome in heart failure patients beyond that available from clinical variables, left ventricular ejection fraction, and b-type natriuretic peptide. ${ }^{1}$ It remains unclear, however, whether further improvement in the prognostic information available from the planar I-123 MIBG H/M ratio can be provided through the use of single-photon emission computed tomography (SPECT). Previously, Chen et al. ${ }^{2}$ compared the accuracy of planar and SPECT imagings for noninvasive quantitation of I-123 MIBG activity in a cardiac phantom model. They noted that SPECT imaging with iterative reconstruction produced more accurate heart-to-calibration ratios than planar imaging. Cardiac SPECT imaging is usually performed with a low-energy, high-resolution (LEHR) collimator. In addition to its predominant $159 \mathrm{keV}$ energy, I-123 has a low abundance, high-energy photon that penetrates the septa of a LEHR collimator, blurring the MIBG images, and impairing the accuracy of quantitative estimates. Those authors had previously reported that deconvolution of septal penetration (DSP) by those high-energy photons combined with iterative reconstruction yielded quantitative estimates of I-123 MIBG activity that were similar to

From the Division of Cardiovascular Diseases, Department of Internal Medicine, University of Cincinnati College of Medicine, Cincinnati, $\mathrm{OH}$.

Reprint requests: Myron C. Gerson, MD, Division of Cardiovascular Diseases, Department of Internal Medicine, University of Cincinnati College of Medicine, P.O. Box 670542, Cincinnati, OH 45267-0542; myron.gerson@uc.edu.

J Nucl Cardiol 2012;19:16-8.

$1071-3581 / \$ 34.00$

Copyright (C) 2011 American Society of Nuclear Cardiology.

doi:10.1007/s12350-011-9495-4 measurements without septal penetration using a mediumenergy all-purpose collimator. ${ }^{2,3}$ This suggests that the combination of iterative reconstruction and DSP might substantially improve quantitation of I-123 MIBG activity in the heart in patient studies. These previous investigations notwithstanding, the I-123 MIBG H/M ratio from SPECT imaging has not entered into common use due to a lack of a standardized and practical method for calculation of the $\mathrm{H} / \mathrm{M}$ ratio, as well as the technical challenges of calculating the $\mathrm{H} / \mathrm{M}$ ratio in heart failure patients with very low uptake of the tracer into the myocardium.

In their study, Chen et al. ${ }^{4}$ describe a new method for calculating the I-123 MIBG H/M ratio from SPECT images. The method was initially developed by comparing its ability to separate abnormal I-123 MIBG H/M ratios in 53 patients with coronary artery disease, prior myocardial infarction, and left ventricular ejection fraction $\leq 40 \%$, from 14 normal volunteers with no history of heart disease. Three versions of the SPECT method (filtered back-projection, iterative reconstruction, and iterative reconstruction with DSP) plus a standard planar H/M method were then tested for their ability to separate 957 heart failure patients from 94 controls from the ADMIRE-HF study. Using receiver operator characteristics (ROC) analysis, the SPECT method with iterative reconstruction and the planar $\mathrm{H} / \mathrm{M}$ ratio method provided comparable separation of heart failure patients from controls. A finding that was likely unexpected is that iterative reconstruction with DSP did not separate patients from controls as effectively as iterative reconstruction alone.

Improved separation of I-123 MIBG activity in the heart from background and scattered activity results in larger values of the $\mathrm{H} / \mathrm{M}$ ratio with the SPECT compared to planar methods. Merlet et al. ${ }^{5}$ provided an early report documenting the high risk for adverse cardiac events in patients with a planar $\mathrm{H} / \mathrm{M}$ ratio less than 1.2. In the ADMIRE-HF study, ${ }^{1}$ a planar value of 1.6 was prospectively tested to separate high-risk from low-risk patients. The value of 1.6 represents two standard deviations below the mean planar $\mathrm{H} / \mathrm{M}$ ratio in normal subjects as reported in the literature. In the present study, the optimal cut-off value for the planar H/M ratio for separating heart failure patients from controls in the validation cohort was 1.58 , and the optimal $\mathrm{H} / \mathrm{M}$ ratio using SPECT imaging with iterative reconstruction was 2.61. This difference likely represents less contamination of the heart region of interest from background 
activity with SPECT compared to planar imaging. It suggests that SPECT imaging has the potential to permit more accurate quantitation of the $\mathrm{H} / \mathrm{M}$ ratio than planar methods.

The method for quantitation of $\mathrm{H} / \mathrm{M}$ ratio from SPECT I-123 MIBG images reported by Chen et al. ${ }^{4}$ is a much needed step forward in the development of cardiac neuronal imaging. The same severe reduction in cardiac I-123 MIBG activity that is highly predictive of adverse cardiac events in heart failure patients also results in great challenges in measurement of radiotracer activity in the myocardium on SPECT images. Anyone who has interpreted I-123 MIBG images, and SPECT MIBG images in particular, knows that accurate delineation of the myocardial borders in a heart failure patient with severely reduced radiotracer activity in the myocardium can be very challenging. The high intra-observer and interobserver agreements for SPECT I-123 MIBG H/M ratio calculation using this new SPECT method is very encouraging. A theoretic advantage of SPECT over planar quantitation of myocardial I-123 MIBG activity is better separation of myocardial from background activity with tomography. This, however, raises the question of whether the myocardium could be more effectively separated from the left ventricular blood pool by an edge-tracking method that accurately traces the myocardial outline excluding the blood pool. With their method, the authors have chosen not to try to exclude the left ventricular cavity from the heart region of interest. This would appear to be a necessary concession given the low I-123 MIBG cardiac count density in patients with severe heart failure.

Potentially valuable information from SPECT imaging of I-123 MIBG activity in the heart is not limited to the $\mathrm{H} / \mathrm{M}$ ratio. Well-validated, reproducible, widely applicable approaches to other measurements from SPECT I-123 MIBG imaging are needed. For example, planar SPECT imaging of I-123 MIBG washout from the myocardium over 3-4 hours has been shown to be predictive of sudden cardiac death, ${ }^{6,7}$ but SPECT approaches to quantitation of I-123 MIBG washout have received limited attention. In patients with an implantable cardioverter-defibrillator (ICD), Boogers et al. ${ }^{8}$ reported that during 3 years of follow-up, 52\% of patients with a large late I-123 MIBG SPECT defect received appropriate ICD therapy compared with $5 \%$ of patients with a small late I-123 MIBG SPECT defect $(P<0.01)$. This underscores the potential need for accurate, reproducible, and generally available software to quantitate I-123 MIBG SPECT defect size. SPECT also offers an important method to quantitate regional myocardial distribution of I-123 MIBG activity. Regional inhomogeneity of I-123 MIBG activity has been reported in patients with increased risk of sudden cardiac death, including arrhythmogenic right ventricular cardio- myopathy, ${ }^{9}$ Brugada syndrome, ${ }^{10,11}$ and Long QT syndrome. ${ }^{12,13}$ Clarification of possible regional differences in sympathetic innervation could be facilitated by accurate quantitation of regional I-123 MIBG distribution using SPECT imaging. It has also been observed that a distribution mismatch of myocardial perfusion and innervation may provide a substrate for life-threatening ventricular arrhythmias. ${ }^{14-16}$ Therefore, SPECT assessment of $\mathrm{H} / \mathrm{M}$ ratio is only one of several potentially important applications of SPECT imaging of cardiac innervation.

It is not clear why the addition of DSP to iterative reconstruction did not contribute positively to the accuracy of SPECT for separating heart failure patients from controls in the present study. Before dismissing the DSP approach completely, it is important to appreciate a fundamental limitation of the present study. It is highly unlikely that I-123 MIBG will find a practical clinical role for separating patients with heart failure (or patients with ischemic heart disease) from control subjects. The promising clinical role for I-123 MIBG relates to its prognostic (rather than diagnostic) capabilities. The clinical outcomes of the heart failure patients in the ADMIRE-HF study are already known. The critically important next steps are to assess whether the iterative reconstruction SPECT method for $\mathrm{H} / \mathrm{M}$ ratio calculation as reported by Chen et al is predictive of adverse cardiac events, whether it predicts events as well or better than the planar method, and to see whether addition of DSP provides any benefit in determining prognosis.

\section{References}

1. Jacobson AF, Senior R, Cerqueira MD, Wong ND, Thomas GS, Lopez VA, et al. Myocardial iodine-123 meta-iodobenzylguanidine imaging and cardiac events in heart failure. Results of the prospective ADMIRE-HF (AdreView Myocardial Imaging for Risk Evaluation in Heart Failure) Study. J Am Coll Cardiol. 2010;55:2212-21

2. Chen J, Garcia EV, Galt JR, Folks RD, Carrio I. Optimized acquisition and processing protocols for I-123 cardiac SPECT imaging. J Nucl Cardiol. 2006;13:251-60.

3. Inoue Y, Suzuki A, Shirouzu I, Machida T, Yoshizawa Y, Akita F, et al. Effect of collimator choice on quantitative assessment of cardiac iodine 123 MIBG uptake. J Nucl Cardiol. 2003;10:623-32.

4. Chen J, Folks RD, Verdes L, Manatunga DN, Jacobson AF, Garcia EV. Quantitative I-123 mIBG SPECT in differentiating abnormal and normal mIBG myocardial uptake. J Nucl Cardiol. doi: 10.1007/s12350-011-9438-0.

5. Merlet P, Benvenuti C, Moyse D, Pouillart F, Dubois-Rande J-L, Duval A-M, et al. Prognostic value of MIBG imaging in idiopathic dilated cardiomyopathy. J Nucl Med. 1999;40:917-23.

6. Tamaki S, Yamada T, Okuyama Y, Morita T, Sanada S, Tsukamoto $\mathrm{Y}$, et al. Cardiac iodine-123 metaiodobenzylguanidine imaging predicts sudden cardiac death independently of left ventricular ejection fraction in patients with chronic heart failure and left ventricular systolic dysfunction. Results from a comparative 
study with signal-averaged electrocardiogram, heart rate variability, and QT dispersion. J Am Coll Cardiol. 2009;53:426-35.

7. Kioka H, Yamada T, Mine T, Morita T, Tsukamoto Y, Tamaki S, et al. Prediction of sudden death in patients with mild-to-moderate chronic heart failure by using cardiac iodine-123 metaiodobenzylguanidine imaging. Heart. 2007;93:1213-8.

8. Boogers MJ, Borleffs CJW, Henneman MM, van Bommel RJ, van Ramshorst J, Boersma E, et al. Cardiac sympathetic denervation assessed with 123-iodine metaiodobenzylguanidine imaging predicts ventricular arrhythmias in implantable cardioverter-defibrillator patients. J Am Coll Cardiol. 2010;55:2769-77.

9. Wichter T, Hindricks G, Lerch H, Bartenstein P, Borggrefe M, Schober O, et al. Regional myocardial sympathetic dysinnervation in arrhythmogenic right ventricular cardiomyopathy. An analysis using ${ }^{123}$ I-meta-iodobenzylguanidine scintigraphy. Circulation. 1994;89:667-83

10. Wichter T, Matheja P, Eckardt L, Kies P, Schafers K, SchulzeBahr E, et al. Cardiac autonomic dysfunction in Brugada syndrome. Circulation. 2002;105:702-6.

11. Agostini D, Scanu P, Loiselet P, Babatasi G, Darlas Y, Grollier G, et al. Iodine-123-metaiodobenzylguanidine SPECT of regional cardiac adrenergic denervation in Brugada syndrome. J Nucl Med. 1998;39:1129-32.
12. Muller KD, Jakob H, Neuzner J, Grebe SF, Schlepper M, Pitschner $\mathrm{HF} .{ }^{123}$ I-metaiodobenzylguanidine scintigraphy in the detection of irregular regional sympathetic innervation in long QT syndrome. Eur Heart J. 1993;14:316-25.

13. Yamanari H, Nakayama K, Morita H, Miyazi K, Fukushima K, Matsubara $\mathrm{H}$, et al. Effects of cardiac sympathetic innervation on regional wall motion abnormality in patients with long QT syndrome. Heart. 2000;83:295-300.

14. Yukinaka M, Nomura M, Ito S, Nakaya Y. Mismatch between myocardial accumulation of ${ }^{123} \mathrm{I}-\mathrm{MIBG}$ and ${ }^{99 \mathrm{~m}} \mathrm{Tc}-\mathrm{MIBI}$ and late ventricular potentials in patients after myocardial infarction: Association with the development of ventricular arrhythmias. Am Heart J. 1998;136:859-67.

15. Sasano T, Abraham MR, Chang K-C, Ashikaga H, Mills KJ, Holt DP, et al. Abnormal sympathetic innervation of viable myocardium and the substrate of ventricular tachycardia after myocardial infarction. J Am Coll Cardiol. 2008;51:2266-75.

16. Calkins H, Allman K, Bolling S, Kirsch M, Wieland D, Morady F, et al. Correlation between scintigraphic evidence of regional sympathetic neuronal dysfunction and ventricular refractoriness in the human heart. Circulation. 1993;88:172-9. 